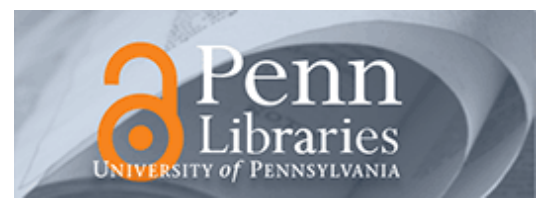

University of Pennsylvania

ScholarlyCommons

August 2007

\title{
Dynamics of electron-trapping materials under blue light and near infrared exposure: an improved model
}

\author{
Ramin Pashaie \\ University of Pennsylvania, raminp@seas.upenn.edu \\ Nabil H. Farhat \\ University of Pennsylvania, farhat@seas.upenn.edu
}

Follow this and additional works at: https://repository.upenn.edu/ese_papers

\section{Recommended Citation}

Ramin Pashaie and Nabil H. Farhat, "Dynamics of electron-trapping materials under blue light and near infrared exposure: an improved model", . August 2007.

Postprint version. Published in Journal of the Optical Society of America, B, Volume 24, Issue 8, August 2007, pages 1927-1941.

This paper was published in Journal of the Optical Society of America, B and is made available as an electronic reprint with the permission of OSA. The paper can be found at the following URL on the OSA website: http://josab.osa.org/abstract.cfm?id=140032. Systematic or multiple reproduction or distribution to multiple locations via electronic or other means is prohibited and is subject to penalties under law.

This paper is posted at ScholarlyCommons. https://repository.upenn.edu/ese_papers/283

For more information, please contact repository@pobox.upenn.edu. 


\title{
Dynamics of electron-trapping materials under blue light and near infrared exposure: an improved model
}

\author{
Abstract \\ Dynamics of electron-trapping materials (ETMs) is investigated. Based on experimental observations, \\ evolution of the ETM's luminescence is mathematically modeled by a nonlinear differential equation. This \\ improved model can predict dynamics of ETM under blue light and near-infrared (NIR) exposures during \\ charging, discharging, simultaneous illumination, and in the equilibrium state. The equilibrium-state \\ luminescence of ETM is used to realize a highly nonlinear optical device with potential applications in \\ nonlinear optical signal processing.

\section{Keywords} \\ fluorescent and luminescent materials, information processing, optical data processing

\section{Comments} \\ Postprint version. Published in Journal of the Optical Society of America, B, Volume 24, Issue 8, August \\ 2007, pages 1927-1941. \\ This paper was published in Journal of the Optical Society of America, B and is made available as an \\ electronic reprint with the permission of OSA. The paper can be found at the following URL on the OSA \\ website: http://josab.osa.org/abstract.cfm?id=140032. Systematic or multiple reproduction or distribution \\ to multiple locations via electronic or other means is prohibited and is subject to penalties under law.
}




\section{Dynamics of electron-trapping materials under}

\section{1. INTRODUCTION}

20 Electron-trapping materials (ETMs) are alkaline-earth 21 sulfides doped with rare-earth luminescence centers [1,2]. 22 Such a material possesses versatile optical properties, in23 cluding high resolution and wavelength diversity, which 24 make it attractive for a variety of technical applications $25[1,2]$. ETM has been employed in the structure of compu26 tational machines such as parallel Boolean logic [3], spa27 tial domain match filtering [4], associative memory [5-7], 28 and adaptive learning [8], as well as optical data storage $29[1,2]$, infrared sensors, image intensifiers, and medium30 wavelength infrared to visible converters $[9,10]$.

31 The atomic structure and the dynamics of ETM under 32 blue light and near-infrared (NIR) illumination have been 33 known for sometime [11], and a few approximate models 34 for the optical mechanism of ETM have been presented $35[12,13]$. The first-order mathematical model that governs 36 the ETM's dynamics was proposed in 1993 [12]. This 37 model contained several restrictions; namely, it assumed 38 the trapped electron density is far from saturation, and 39 the effect of interaction between blue photons and the 40 trapped electrons is negligible. Furthermore, in this 41 model, electron-trapping efficiency was assumed to be in42 dependent of the current level of the trapped electron 43 density. With these simplifications, the charging and dis44 charging processes were modeled by linear and exponen45 tial functions, respectively.

46 A modified version of this early model was presented in 471995 [13] that took into account some of the previously 48 neglected effects. In this modified model, the intensity of 49 the ETM's luminescence was a function of the intensity of 50 both the charging blue light and the discharging NIR ex51 posures. Also, the saturation in the density of trapped 52 electrons was considered in the equations. Both charging and discharging processes were exponential. Nevertheless, predictions of the model do not agree well with ex- 54 perimental results.

Extending the utility of ETMs to quantitative applica- 56 tions requires a mathematical model that predicts its 57 unique dynamics precisely. In this paper, we present an 58 improved model that can simulate the evolution of ETM's 59 luminescence during charging and discharging, including 60 simultaneous blue light and NIR exposure, and in the 61 equilibrium state. Predictions of the model agree with ex- 62 perimental observations.

In Section 2 we briefly review the physics and the opti- 64 cal mechanism of ETM. The chain of experiments that led 65 us to the improved model are detailed in Section 3, and 66 the mathematical model is presented in Section 4. Section 67 5 is devoted to the equilibrium-state luminescence of 68 ETM. We show how the equilibrium state of an ETM can 69 be used to conceive nonlinear optical devices. Concluding 70 remarks are given in Section 6.

\section{DYNAMICS OF ETM UNDER BLUE LIGHT 72 AND NEAR-INFRARED ILLUMINATION 73}

The ETM used in this study is $\mathrm{SrS}: \mathrm{Eu}^{2+}, \mathrm{Sm}^{3+}$, which is 74 basically wide-bandgap strontium sulfides (SrS) doped 75 with two types of selected rare-earth elements, $\mathrm{Eu}^{2+}$ and 76 $\mathrm{Sm}^{3+}$. Fabrication of relatively large-area thin films of 77 this ETM is easy. The atomic structure and the optical 78 mechanism of ETM under simultaneous blue light and 79 NIR exposure are depicted in Fig. 1. Both of the two rare- 80 earth doping elements have ground and excited states 81 within the wide bandgap of the host; however, the lumi- 82 nescence centers of $\mathrm{Eu}^{2+}$ can easily give up an electron to 83 become $\mathrm{Eu}^{3+}$, and the electron-trapping centers of the 84 


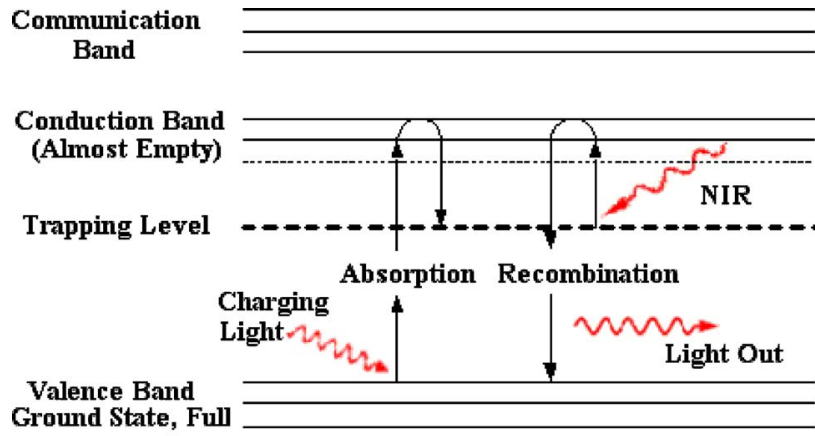

Color: Fig. 1. (Color online) Optical mechanism of charging and disOnline charging of ETM. Interaction of blue photons and electrons of the valence band excites the electrons and sends them to the communication band. Those excited electrons will tunnel to the trap level and become trapped electrons. Infrared photons give sufficient energy to the trapped electrons to detrap and excite them to the communication energy level. These electrons release their extra energy in the form of orange luminescence during their return to the valence band.

${ }^{85} \mathrm{Sm}^{3+}$ can easily accept an electron to become $\mathrm{Sm}^{2+}$. $\mathrm{Ab}$ 86 sorption of blue light with a peak response at $\approx 450 \mathrm{~nm}$ 87 excites electrons from the ground state to the excited 88 state of $\mathrm{Eu}^{2+}$ ions. Once in the excited state, electrons 89 tunnel to neighboring $\mathrm{Sm}^{3+}$ sites and then fall to the 90 ground state to become trapped electrons, leaving behind $91 \mathrm{Eu}^{3+}$ and $\mathrm{Sm}^{2+}$ ions.

92 Blue photons can also provide sufficient energy to kick 93 the electrons from the trap level to the communication 94 level, where they may interact and return to the ground 95 state accompanied by the emission of photons with peak 96 response at $\approx 640 \mathrm{~nm}$ (orange light luminescence). How97 ever, when the density of trapped electrons is much lower 98 than the density of electrons in the valence band, the 99 probability of interaction between blue photons and elec100 trons of the valence band is higher than having such an 101 interaction with trapped electrons. If blue light illumina102 tion persists for a while, a greater number of electrons 103 will be trapped, which increases their chance of interact104 ing with blue photons. As a result, if an ETM with empty 105 trap levels is exposed to blue light illumination, the inten106 sity of the orange light emission will reach the saturation level after a sharp growth. The intensity of orange light emission during blue light exposure can be used as a means of estimating the density of trapped electrons.

When the blue light is removed, the information car- 110 ried in by the blue light pattern is stored in the ETM as a 111 trapped electron density distribution. If the charged ETM 112 is exposed to uniform NIR light with peak response at 113 $\approx 1310 \mathrm{~nm}$, sufficient energy is provided that the trapped 114 electrons are excited from the ground state of $\mathrm{Sm}^{2+}$ to the 115 excited state and tunnel back to neighboring $\mathrm{Eu}^{3+}$ sites. 116 Again, subsequent relaxation from the $\mathrm{Eu}^{3+}$ excited state 117 to the ground state produces emission of orange light. Un- 118 der simultaneous illumination of the ETM by constant 119 blue light and NIR exposure, after a transient response, 120 orange emission converges to a constant value that is the equilibrium-state luminescence of the ETM.

Since the mechanism involved is purely electronic, is purely electronic, ETMs are fast, possessing nanosecond response times un- 124 der both blue light and infrared illuminations. They pro- 125 vide long-term storage of information when kept dark and 126 have potential for high-density storage $(>100 \mathrm{lp} / \mathrm{mm}) \quad 127$ when fabricated in crystalline thin film form [14].

\section{EXPERIMENTS}

An ordered sequence of experiments has been carried out 130 to verify the dynamics of ETM with the experimental 131 setup illustrated in Fig. 2. A thin layer of electron- 132 trapping material deposited on a $25 \mathrm{~mm} \times 25 \mathrm{~mm}$ layer of 133 quartz [15] is exposed to two different sources of light: a 134 bright blue LED and an Exalos $20 \mathrm{~mW}$ fiber coupled 135 $1310 \mathrm{~nm}$ NIR superluminescence laser diode. A center- 136 hole dark mask is placed on the ETM panel. This mask 137 defines the area of the ETM under illumination in the fol- 138 lowing experiments. A blue optical filter blocks possible 139 infrared radiation of the blue LED. Drivers of both light 140 sources are precisely controlled by digital-to-analog con- 141 verters (DACs) of a microcontroller board. An orange op- 142 tical filter (Semrock LP01-633Rs-25) is placed in front of a 143 cooled avalanche photodiode (APD) module (Hamamatsu 144 CA4777-01) to measure the orange luminescence of the 145 ETM.

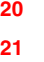

\section{.}

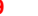

\section{.}

\section{.}

\section{(n)} ${ }_{122}^{22}$ 23 24

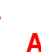

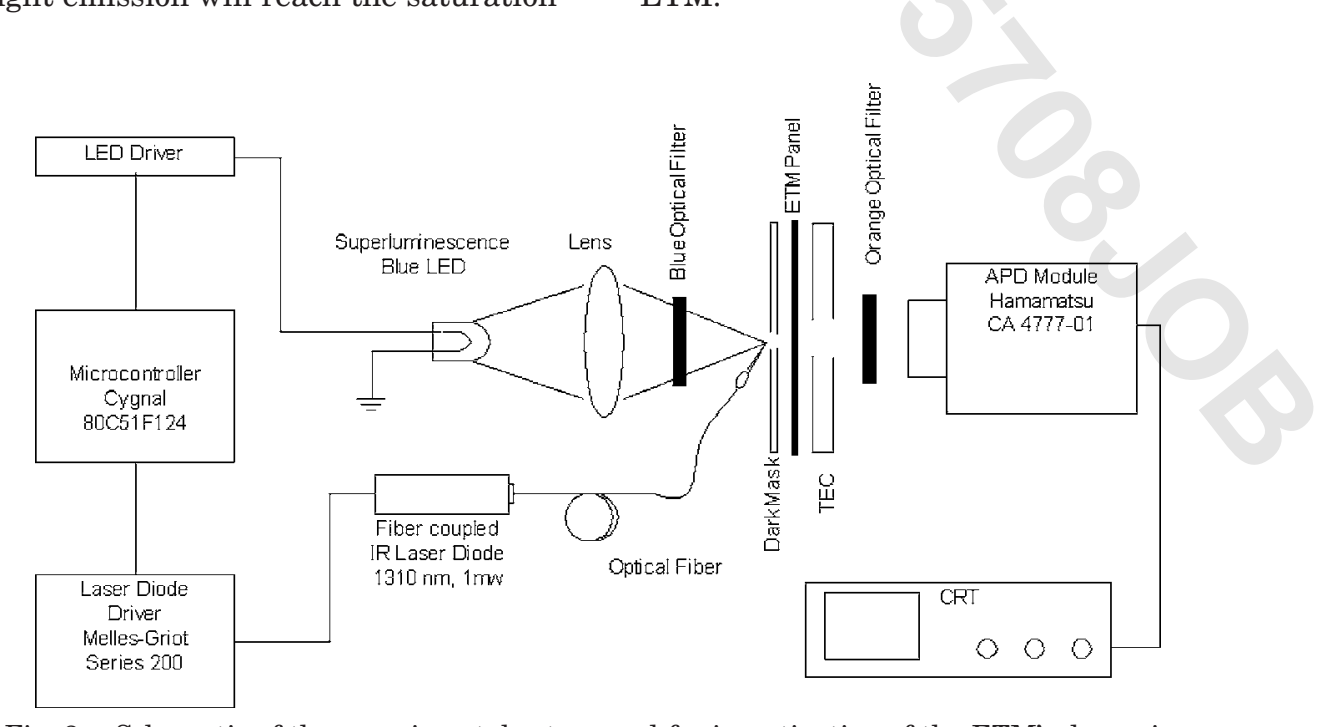

Fig. 2. Schematic of the experimental setup used for investigation of the ETM's dynamics. 


\section{PROOF COPY [78556] 545708JOB}

R. Pashaie and N. H. Farhat

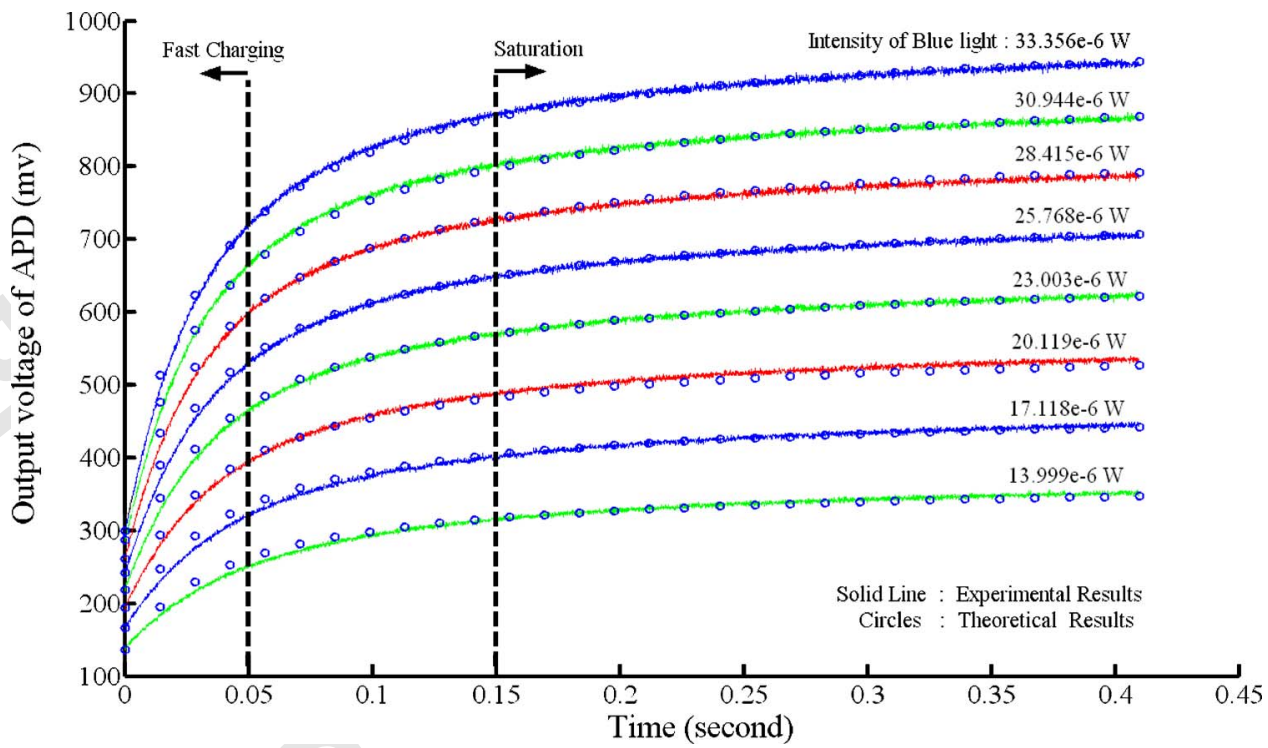

Fig. 3. (Color online) Charging characteristic curves of the partially erased ETM under blue light illumination. Three points should be considered in the study of these curves: the initial jumps (at $t=0.0$ ), the relatively linear buildup in the initial moments, and the final saturations. The solid curves are the experimental results, and the circles are the curve-fitting data.

147 The output voltage of the APD module is connected to 148 the analog input terminal of the microcontroller's analog149 to-digital converter (ADC). A center-hole thermoelectric 150 cooler (TEC) is thermally coupled to the quartz substrate. 151 Cooling the ETM decreases the chance of electron-phonon 152 interaction and the self-luminescence of the phosphor. 153 Also, it has been shown that the orange light emission of 154 the ETM increases by decreasing the temperature of the 155 material [16]. Therefore, the TEC improves the signal-to156 noise ratio.

157 In the first experiment, we investigate the charging 158 process of the ETM under blue light illumination. At the 159 beginning, the blue LED is off when the ETM is exposed 160 to the NIR laser, and the APD module measures the in161 tensity of the orange light emission. When the output 162 voltage of the APD reaches a constant predefined value, 163 the microcontroller's DAC turns off the NIR laser and turns on the blue LED with a specified electric current. Next, the microcontroller's ADC digitizes and records the output voltage of the APD for a fraction of a second. We repeat a similar protocol sequentially with different 167 blue light intensities. Figure 3 displays the charging char- 168 acteristic curves developed. Three key points should be 169 noted in the study of the results obtained: the initial jump 170 in the intensity of the emitted orange light at the begin- 171 ning of the blue light illumination, its relatively linear be- 172 havior during the initial few milliseconds, and the final saturation.

The existence of different saturation levels can be justified by considering that blue photons have sufficient energy not only to excite the electrons of the valence band and send them to the trap level, but also to detrap some of the trapped electrons. The final saturation represents the equilibrium state of these two opposing processes. The n 173 174 ,

\section{.}

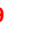

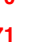

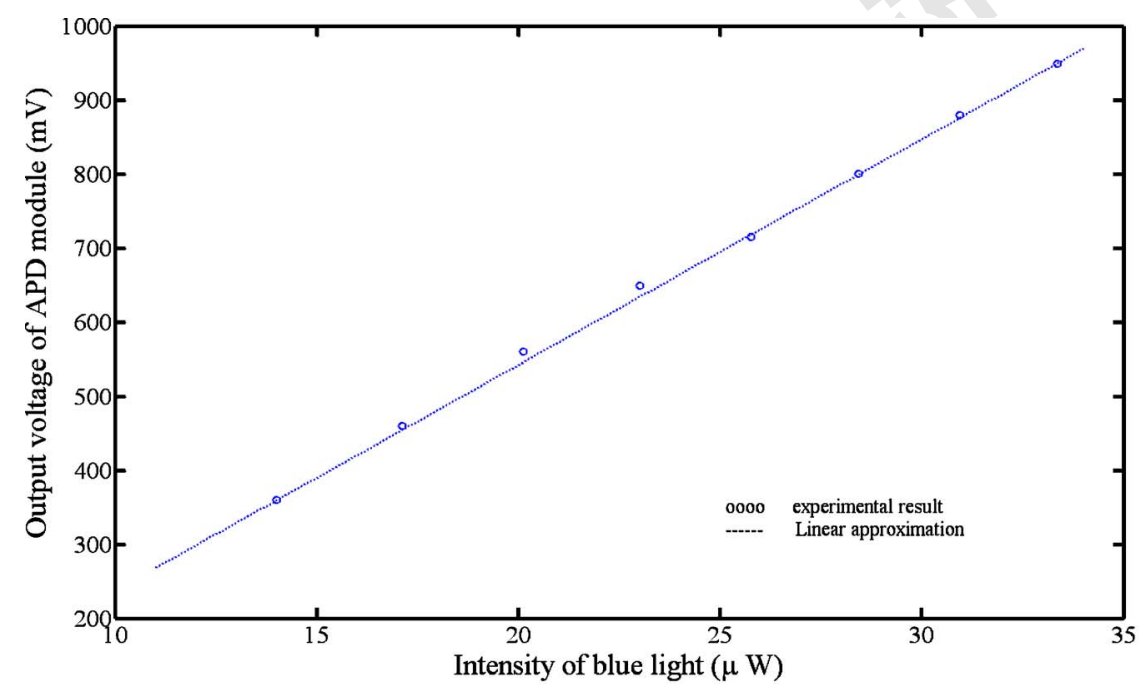

Fig. 4. (Color online) Saturation levels of the partially erased ETM as a function of the charging blue light intensity and the corresponding linear approximation. 


\section{PROOF COPY [78556] 545708JOB}

${ }^{181}$ level of saturation is a function of the blue light intensity. 182 This dependency can be approximated by a linear func183 tion as shown in Fig. 4. Although the ETM is primarily 184 erased to the same density of trapped electrons before all 185 the charging periods, each curve has a different initial 186 jump. This observation suggests that under blue light il187 lumination the intensity of orange light luminescence is a 188 function of both the trapped-electron density and the in189 tensity of the incident blue light.

190 The saturation level of the orange light emission is one 191 of the ETM's critical parameters in the design of nonlin192 ear optical devices (as will be described later) and it needs 193 to be investigated further. From the charging curves of 194 Fig. 3, it is apparent that the saturation level is a function 195 of the blue light intensity. However, these curves were de196 veloped by charging an ETM that was primarily dis197 charged to a constant predefined trapped-electron density. 198 Hence, an unanswered question remains: Does the inten199 sity of orange luminescence reach the same saturation 200 level when the charging process, with constant blue light 201 intensity, starts from different trapped-electron densities?
To answer this question, a series of experiments was performed as follows. First, the precharged ETM is ex- 203 posed to an intense NIR light while the APD module mea- 204 sures the level of the orange light emission. Next, for a 205 fraction of a second, the partially discharged ETM is illu- 206 minated by a constant blue light. Simultaneously, the mi- 207 crocontroller's ADC digitizes and records the intensity of 208 the orange light luminescence. We repeat this experiment 209 with a similar protocol, but in each trial, the NIR light is 210 turned off at different values of the APD output voltage. 211 Thus, the charging process in each trial starts from a dif- 212 ferent density of trapped electrons. The curves developed 213 in this process are displayed in Fig. 5. The curves of Fig. 214 5(b) are similar to the ones illustrated in Fig. 5(a) with 215 more emphasis on initial moments. The experimental re- 216 sults in Fig. 5(a) show that the final saturation level is in- 217 dependent of the initial trapped-electron density. The 218 curves of Fig. 5(b) illustrate that the slope of the charging 219 curve depends on the initial conditions. The charging 220 curves that start from a higher density of the trapped 221 electrons have larger slopes compared with the curves 222

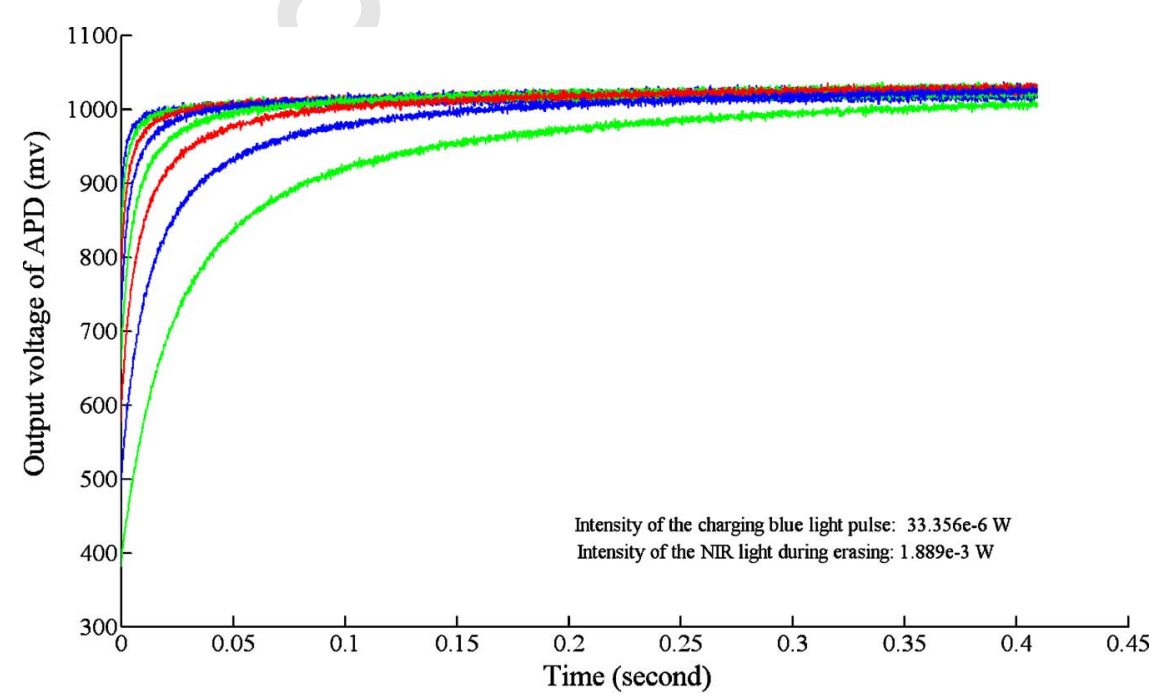

(a)

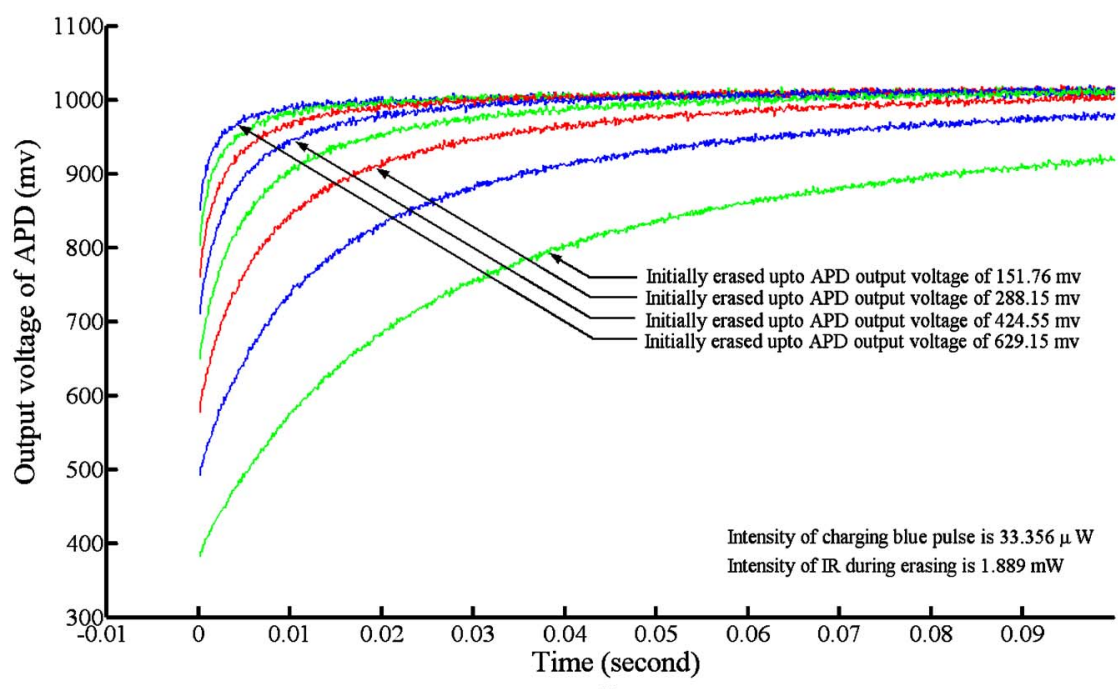

(b)

Fig. 5. (Color online) Charging of the partially erased ETM by constant blue light illumination. (a) Despite different initial density of trapped electrons, all the curves merge to the same saturation level. (b) Different initial jumps under constant blue light illumination. 
223 starting from a lower trapped-electron density. The ETM 224 charges faster when the charging process starts with a 225 higher density of trapped electrons. This observation will 226 be used later in the design of a nonlinear optical device.

227 Figure 6(a) displays the levels of the initial jumps as a 228 function of the intensity of the blue light when the initial 229 density of the trapped electrons is constant. It is clear 230 that the experimental data can be approximated by a lin231 ear function. The graph of Fig. 6(b) illustrates the levels 232 of the initial jumps as a function of the initial trapped233 electron density, when the intensity of the incident charg234 ing blue light is constant. Again, the experimental data 235 developed can be approximated by a linear function. By 236 investigating the data developed from these experiments, 237 the relation between the intensity of the orange lumines238 cence and the intensity of the charging blue light can be 239 formulated. The intensity of the orange light emission is a 240 linear function of the blue light intensity when the charg241 ing process starts from equal initial densities of trapped electrons. The intensity of the orange luminescence is also a linear function of the density of the trapped electrons during the charging process with constant blue light intensity. Consequently, the intensity of the orange light 245 emission during the charging process is proportional to 246 the product of the current value of the trapped-electron 247 density and the intensity of the blue light.

The same experimental setup is used to extract the dis- 249 charging curves of ETM. We perform this experiment in 250 three sequential steps. First, the ETM is exposed to in- 251 tense NIR illumination that detraps almost all the 252 trapped electrons. In the second step, the ETM is exposed 253 to a pulse of blue light with a constant intensity and du- 254 ration. In the third and last step, the ETM panel is illu- 255 minated by a constant NIR light while the intensity of the 256 emitted orange light is digitized and recorded. Then, the 257 same protocol is repeated by changing the intensity of the 258 NIR light in the last step of the experiment. The group of 259 curves displayed in Fig. 7 represents the results of the 260

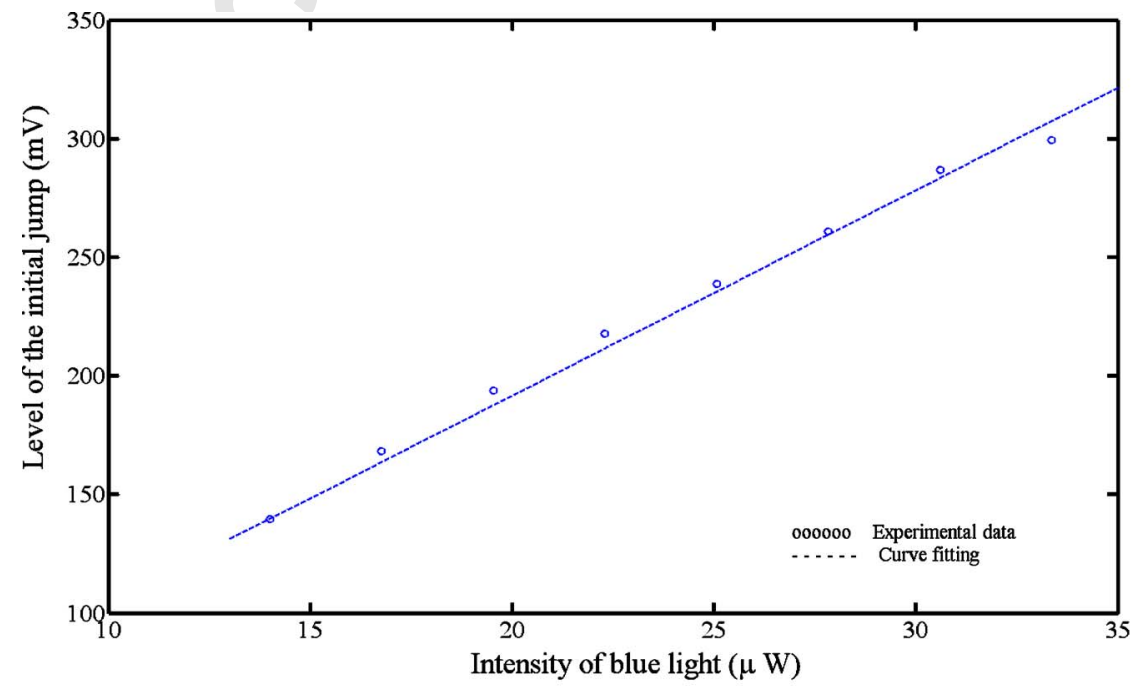

(a)

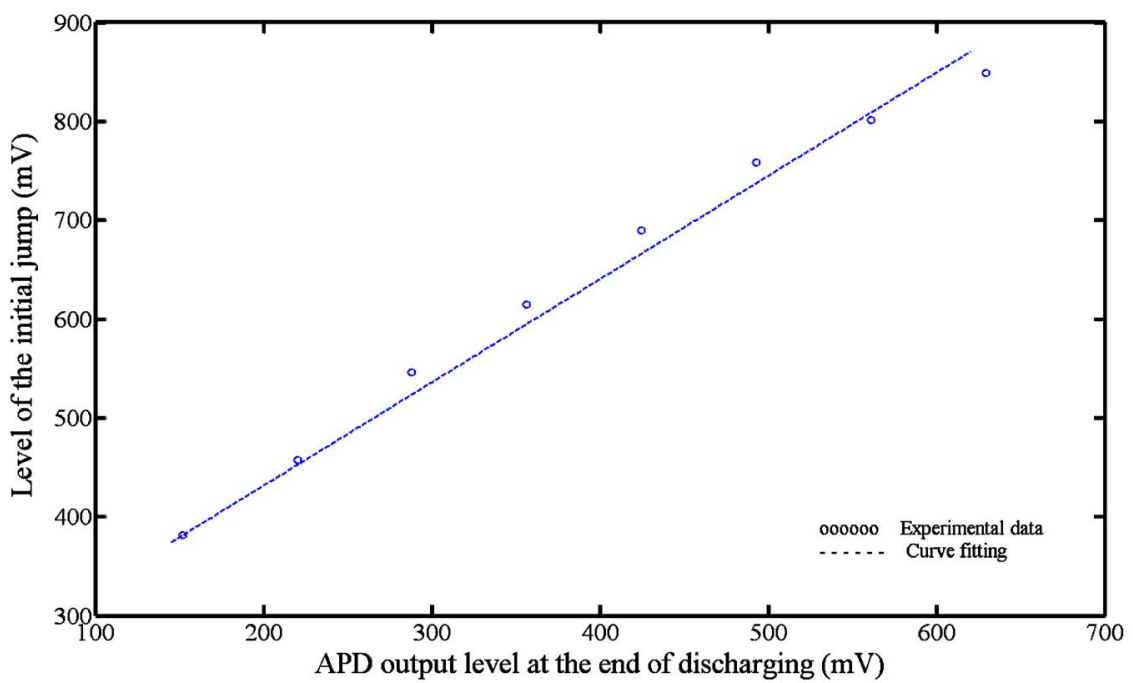

(b)

Fig. 6. (Color online) (a) Levels of the initial jumps as a function of the intensity of the blue light when the initial density of the trapped electrons is constant. The experimental data can be approximated by a linear function. (b) The levels of the initial jumps as a function of the initial trapped-electron density when the intensity of the incident charging blue light is constant. Again, the experimental data can be approximated by a linear function. 


\section{PROOF COPY [78556] 545708JOB}

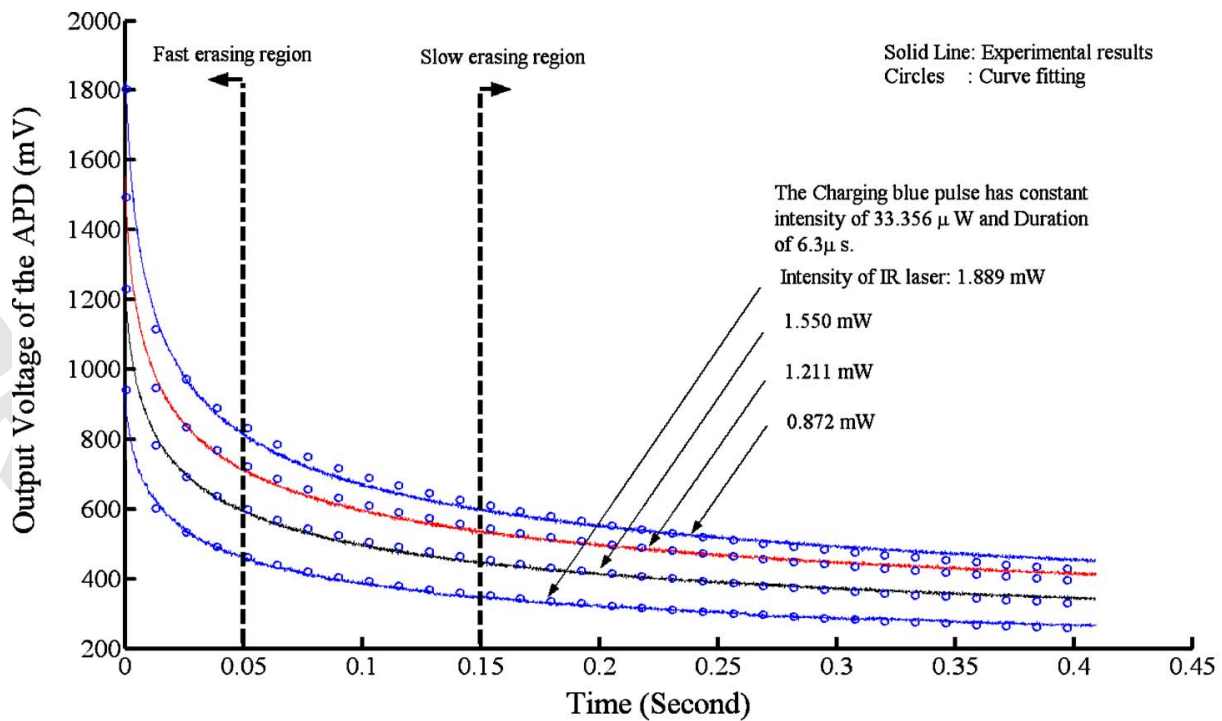

Fig. 7. (Color online) Discharging characteristic curves of the ETM. The discharging process has two separable phases. In the first phase, the intensity of the orange light emission drops rapidly after an abrupt jump. During the second phase, the intensity of the orange light emission decreases slowly.

${ }^{261}$ discharging experiment. These curves show that the dis262 charging process has two different phases. During the 263 first phase, which occurs at the initial moment, the inten264 sity of the emitted orange light drops rapidly and the dis265 charging process is even faster than the exponential func266 tion. The initial jumps and the slopes of the curves in this 267 figure are functions of the initial density of the trapped 268 electrons and the intensity of the NIR exposure, the 269 higher the initial density of trapped electrons, the steeper 270 the discharging process. During the second phase, the or271 ange light emission decreases slowly and the discharging 272 process is slow. Figure 8 displays the levels of the initial 273 jumps as a function of the discharging NIR light intensity. 274 The experimental results in this graph can be approxi275 mated by a linear function. Hence, as with the charging 276 process, the intensity of the orange light emission during 277 discharging is proportional to the product of the intensity of the NIR light and the existing value of the trappedelectron density.

278

ates the effect of temperature on the intensity of the orange light emission. In this experiment, 281 the ETM panel is simultaneously exposed to the blue light 282 and the NIR laser, and the equilibrium-state emission of 283 the ETM is recorded under different TEC currents. This 284 experiment elucidates that increasing the current of the 285 TEC (which is equivalent to reducing the temperature of 286 ETM) increases the intensity of the emitted orange light 287 and improves the corresponding signal-to-noise ratio of 288 the APD module output voltage. In practice, by cooling 289 the ETM, detectors with lower sensitivity that are 290 cheaper and available in array formats can be employed. 291 This could become a critical issue during realization of 292 multipixel systems. The temperature dependence of the 293 photoluminescence in the singly doped ETMs $\left(\mathrm{SrS}: \mathrm{Em}^{2+} 294\right.$

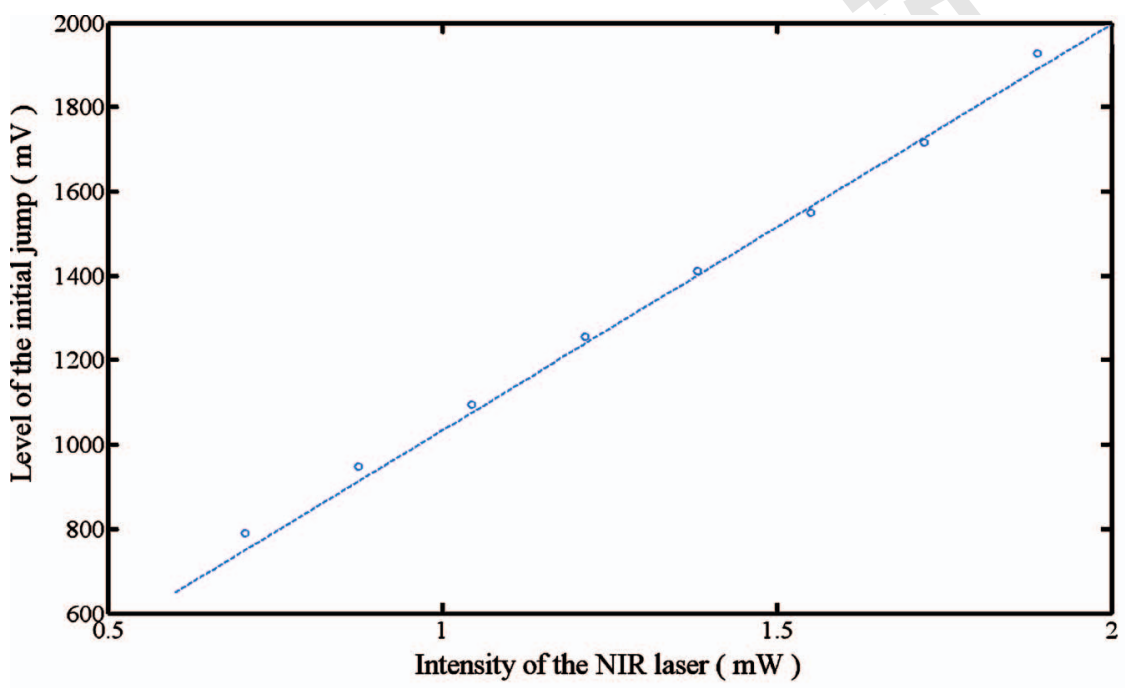

Fig. 8. Levels of the initial jumps as a function of the discharging NIR light intensity. A linear function can be fitted to the experimental results. 
PROOF COPY [78556] 545708JOB

R. Pashaie and N. H. Farhat

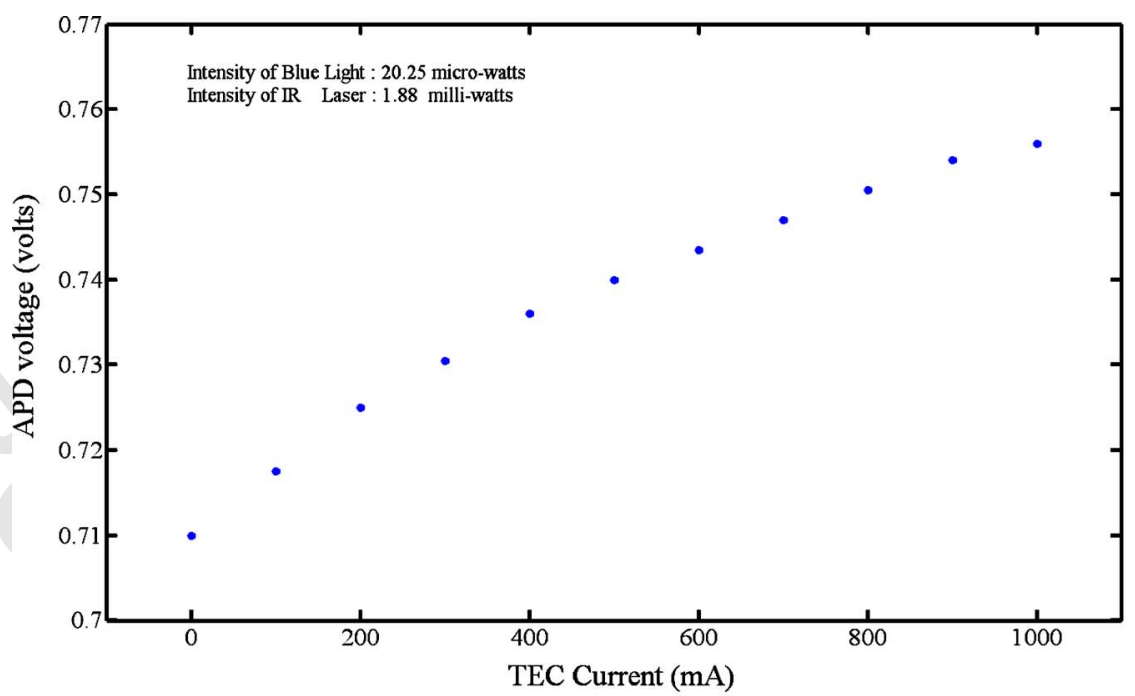

Color:

Fig. 9. (Color online) Effect of temperature on the intensity of the emitted orange light when the ETM is under simultaneous blue light and NIR light illumination. This curve proves that at lower temperatures, the orange light emission is more intense.

${ }^{295}$ and $\mathrm{SrS}: \mathrm{Sm}^{3+}$ ) as well as the doubly doped ones (e.g. $296 \mathrm{SrS}: \mathrm{Em}^{2+}, \mathrm{Sm}^{3+}$ ) have been extensively studied [16].

\section{4. MATHEMATICAL MODEL}

298 In this section, the dynamics of the ETM under simulta299 neous blue light and NIR light illumination is cast in the 300 form of a nonlinear differential equation based on the ex301 perimental results discussed in Section 3.

302 Suppose that $n(t)$ represents the temporal density of 303 the trapped electrons as a function of time $t$. During the 304 charging period when ETM is exposed just to the blue 305 light, the density of the trapped electrons $n(t)$ increases 306 monotonically from the initial density of the trapped elec- trons $n(0)$ to the saturation density $n_{s}$ where $0 \leqslant n(t)$ $\leqslant n_{s}$. By optimal curve fitting [17], the charging process 308 can be formulated as

$$
n(t)=n_{s}\left(I_{\mathrm{B}}\right)-\xi I_{\mathrm{B}} L n\left(\frac{\eta}{t+t_{s}}+1\right) .
$$

The saturation density of the trapped electrons is a func- 311 tion of the intensity of the incident blue light $I_{\mathrm{B}}$. Follow- 312 ing our experimental results (Fig. 4), the dependency of 313 the $n_{s}$ on the intensity of the charging blue light can be 314 approximated by a linear function such as $n_{s}\left(I_{\mathrm{B}}\right)=\kappa I_{\mathrm{B}} \quad 315$ where $\kappa$ is a real constant.

During the discharging period when ETM is exposed to 317 the NIR light, the density of the trapped electrons mono- 318

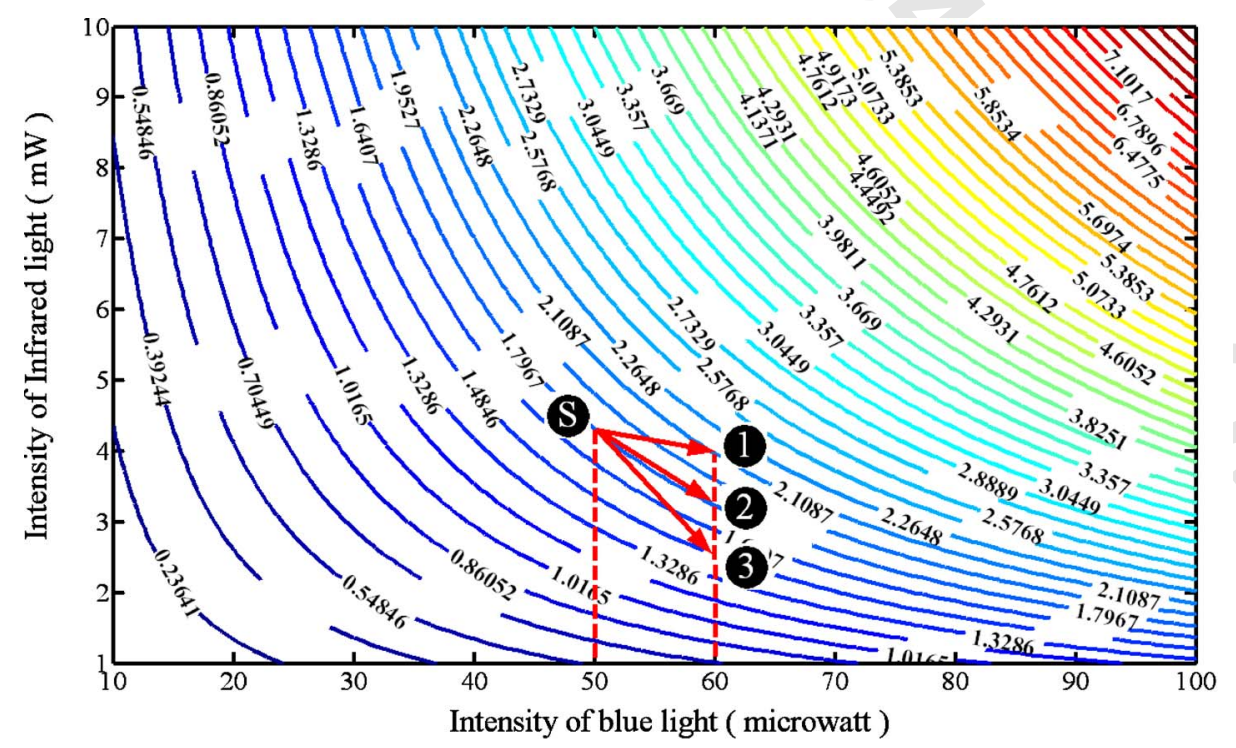

AQ: $\quad$ Fig. 10. (Color online) ESP of electron-trapping material and the unexpected behavior of the ETM luminescence in the equilibrium state. 


\section{PROOF COPY [78556] 545708JOB}

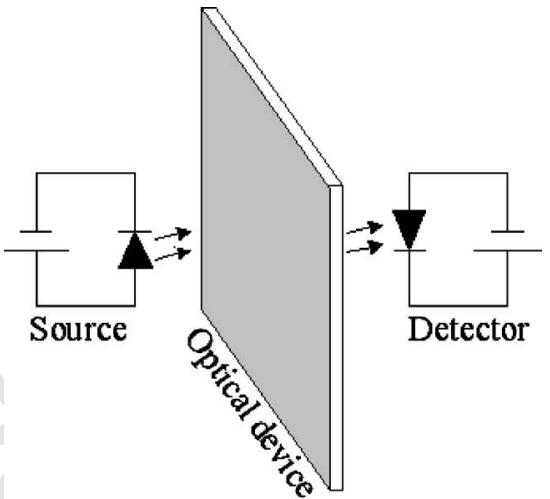

(a)

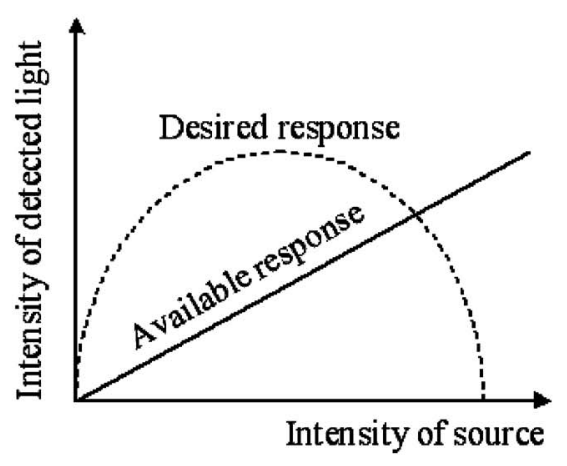

(b)

Fig. 11. (a) Optical setup. A light source illuminates the optical device and a detector measures the intensity of the light that passes through the optical device. (b) Available and desired response curves.

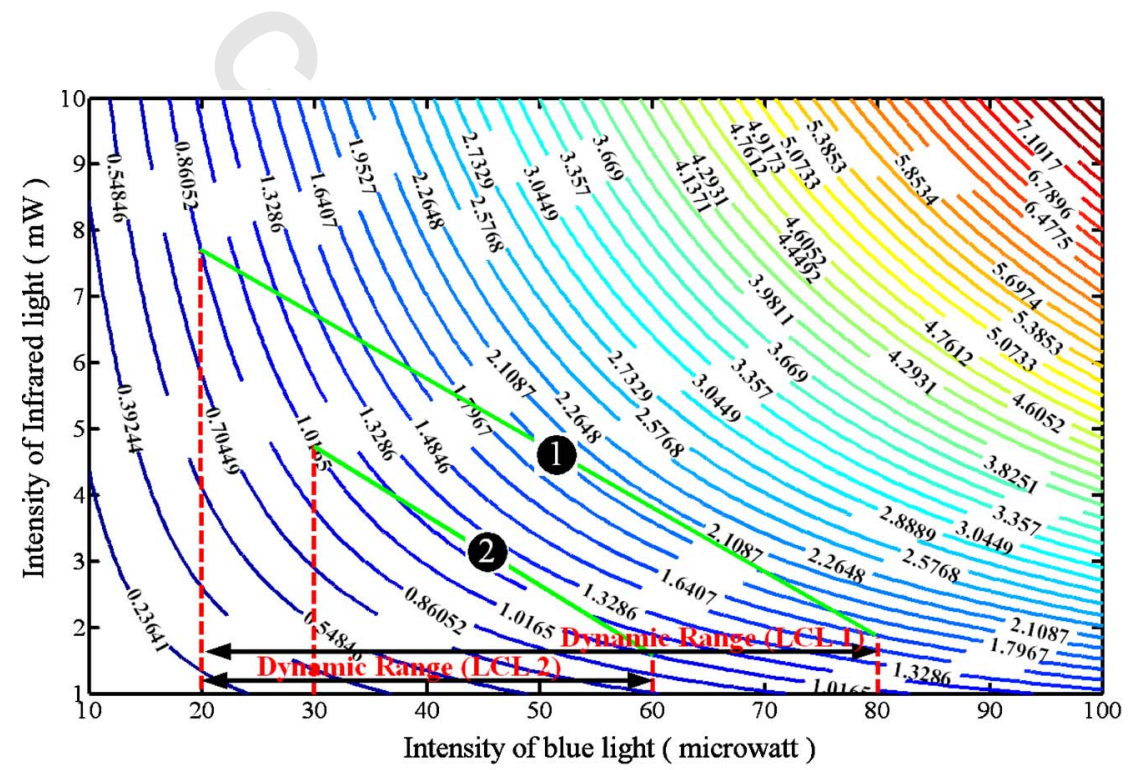

(a)

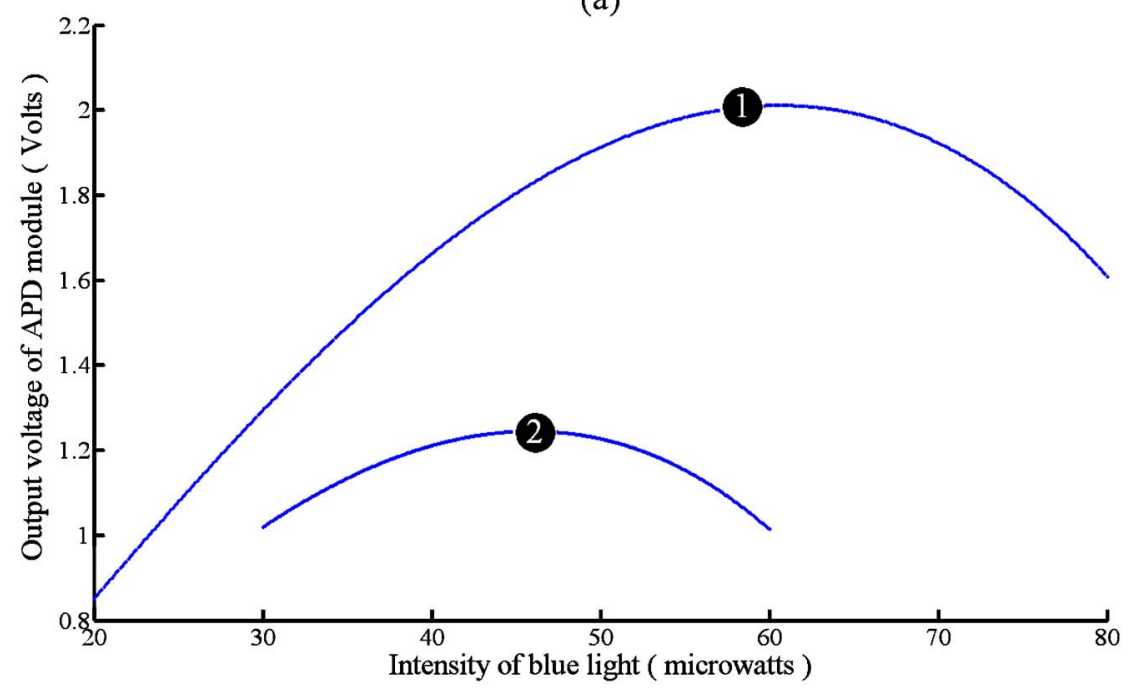

(b)

Color: Fig. 12. (Color online) (a) Two sample LCL and their dynamic ranges along the blue light intensity axis in the ESP of the ETM. Only Online the intensities of the emitted orange light along the LCLs are accessible when the light sources are linearly coupled. (b) The corresponding nonlinear curves. 
319 tonically decreases. As with the charging process, the dis320 charging process is formulated by optimal curve fitting 321 [17] as

322

$$
n(t)=\xi^{\prime} I_{\mathrm{NIR}} \operatorname{Ln}\left(\frac{\eta^{\prime}}{t+t_{s^{\prime}}}+1\right)
$$

323 where $I_{\mathrm{NIR}}$ is the intensity of the NIR light. In Eqs. (1) 324 and (2), $\xi$ and $\xi^{\prime}$ are the ETM wavelength-dependent sen325 sitivity coefficients to the blue light and the NIR light ex326 posures. Variables $\eta$ and $\eta^{\prime}$ are the curve fitting param327 eters, and the initial jumps during the charging and the 328 discharging processes are modeled by the variables $t_{s}$ and $329 t_{s^{\prime}}$, respectively. Obviously, $t_{s}$ and $t_{s^{\prime}}$ are functions of the 330 initial density of the trapped electrons $n(0)$ and the inten331 sity of the blue light and NIR exposure. The curve fittings 332 of the experimentally derived data during charging and 333 discharging processes are displayed in Figs. 3 and 7.

334 In Section 3 it was experimentally proved that $I_{\mathrm{O}}$, the 335 intensity of the orange light emission under simultaneous 336 blue light and NIR illumination, can be expressed as

$$
I_{\mathrm{O}}(t)=\alpha n(t) I_{\mathrm{B}}+\beta n(t) I_{\mathrm{NIR}}
$$

337

where $\alpha$ and $\beta$ are the proportionality coefficients for the 338 blue light and the NIR light intensities. Usually, we mea- 339 sure the intensity of the orange luminescence by a photo- 340 detector. The output voltage of this transducer $V_{\mathrm{O}}$ is pro- 341 portional to the illuminating optical intensity: $V_{\mathrm{O}} \propto I_{\mathrm{O}} . \quad 342$ From Eq. (1), the growth rate of the trapped-electron den- 343 sity is

$$
\dot{n}=\frac{\mathrm{d} n}{\mathrm{~d} t}=\frac{\xi I_{\mathrm{B}}}{\eta} \frac{\left[\exp \left(\frac{n_{s}-n}{\xi I_{\mathrm{B}}}\right)-1\right]^{2}}{\exp \left(\frac{n_{s}-n}{\xi I_{\mathrm{B}}}\right)} .
$$

Equation (1) can also be rearranged in the form

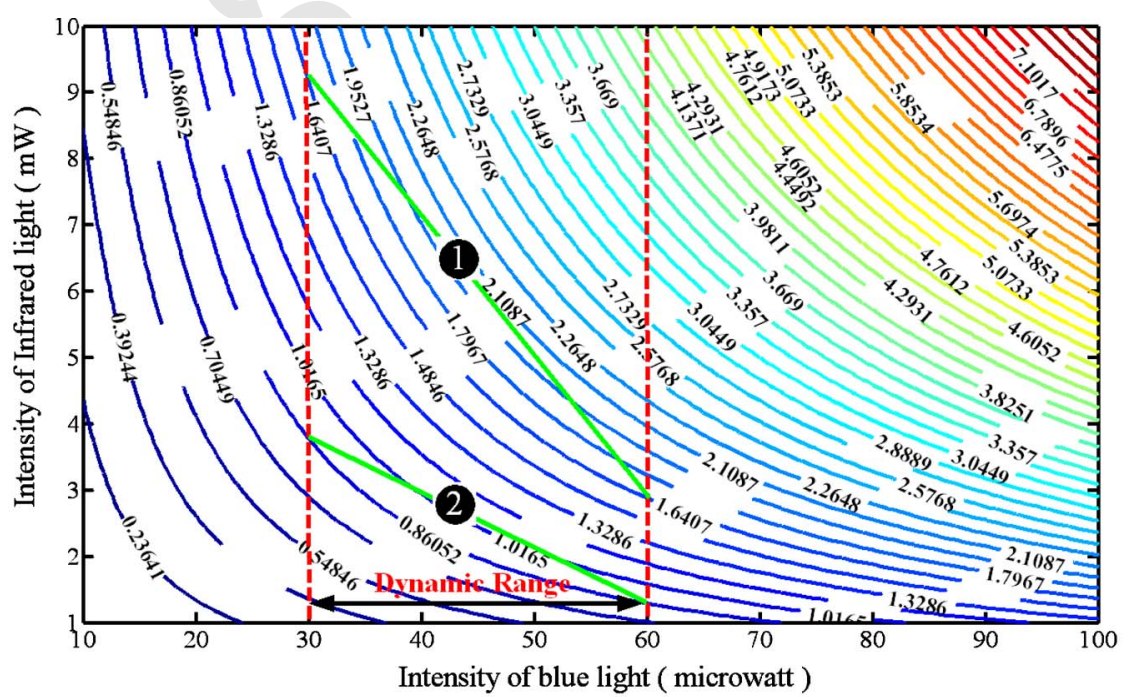

(a)

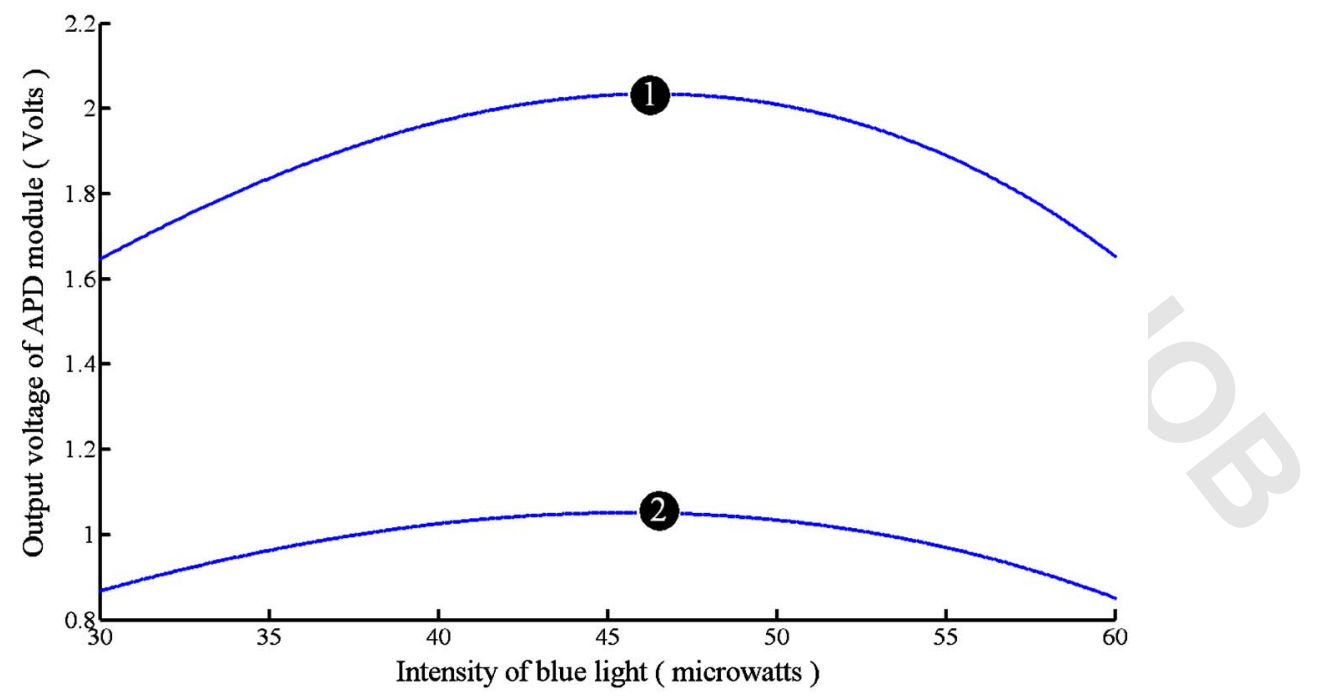

(b)

Fig. 13. (Color online) (a) Two LCLs with the same dynamic range. The termination points of each of these LCLs are located on the same contour. (b) The intensities of the emitted orange light along two LCLs that are depicted in Fig. 14(a). 


\section{PROOF COPY [78556] 545708JOB}

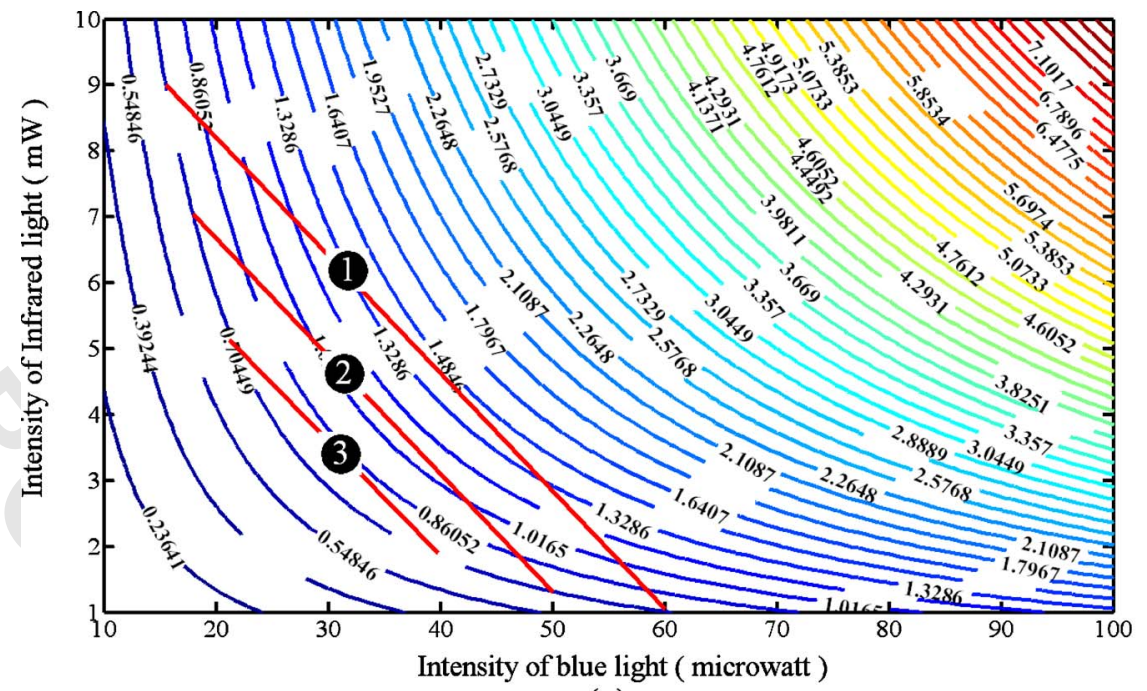

(a)

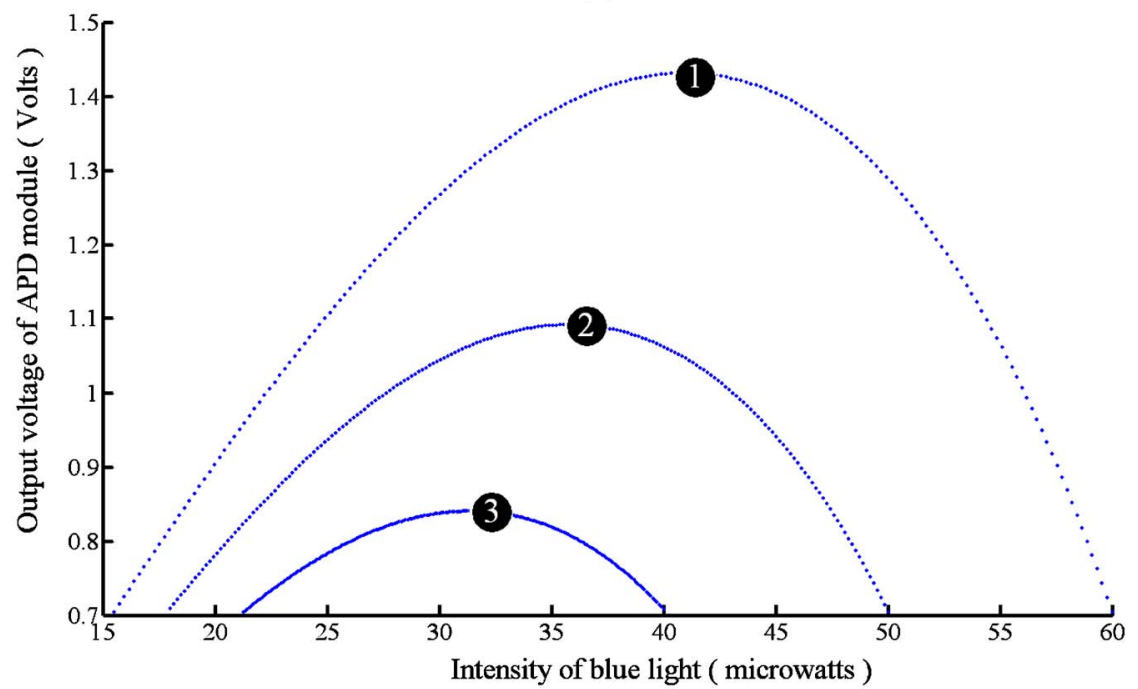

(b)

Fig. 14. (Color online) (a) Parallel LCLs. All the terminating points are on the same contour. (b) The crresponding nonlinear curves. This type of nonlinear curve can be used in the optical generation of one-dimensional maps.

347

$$
\frac{\eta}{t+t_{s}}=\exp \left(\frac{n_{s}-n}{\xi I_{\mathrm{B}}}\right)-1 .
$$

348 Similarly, using Eq. (2), the reduction rate of the trapped349 electron density during the discharging process is

350

$$
\dot{n}=\frac{\mathrm{d} n}{\mathrm{~d} t}=\frac{\xi^{\prime} I_{\mathrm{NIR}}}{\eta^{\prime}} \frac{\left[\exp \left(\frac{n}{\xi^{\prime} I_{\mathrm{NIR}}}\right)-1\right]^{2}}{\exp \left(\frac{n}{\xi^{\prime} I_{\mathrm{NIR}}}\right)},
$$

351 and by rearranging Eq. (2),

352

$$
\frac{\eta^{\prime}}{t+t_{s}^{\prime}}=\exp \left(\frac{n}{\xi^{\prime} I_{\mathrm{NIR}}}\right)-1 \text {. }
$$

353 Equations (5) and (7) can be solved for the parameters $t_{s}$ 354 and $t_{s^{\prime}}$ :

$$
\begin{gathered}
t_{s}=\frac{\eta}{\exp \left(\frac{n_{s}-n}{\xi I_{\mathrm{B}}}\right)-1}, \\
t_{s^{\prime}}=\frac{\eta^{\prime}}{\exp \left(\frac{n}{\xi^{\prime} I_{\mathrm{NIR}}}\right)-1} .
\end{gathered}
$$

Now, Eqs. (4) and (6) can also be rewritten in the forms 357

$$
\begin{aligned}
& \dot{n}=\frac{4 \xi}{\eta} I_{\mathrm{B}} \sinh ^{2}\left(\frac{n_{s}-n}{2 \xi I_{\mathrm{B}}}\right), \\
& \dot{n}=-\frac{4 \xi^{\prime}}{\eta^{\prime}} I_{\mathrm{NIR}} \sinh ^{2}\left(\frac{n}{2 \xi^{\prime} I_{\mathrm{NIR}}}\right) .
\end{aligned}
$$

The mathematical model for the charging and discharg- 360 ing processes is expressed in Eqs. (10) and (11). By com- 361 
${ }^{362}$ bining these two expressions, we develop the mathemati$363 \mathrm{cal}$ model that governs the dynamics of the ETM under 364 simultaneous blue light and NIR illumination:

$$
\dot{n}=\frac{4 \xi}{\eta} I_{\mathrm{B}} \sinh ^{2}\left(\frac{n_{s}-n}{2 \xi I_{\mathrm{B}}}\right)-\frac{4 \xi^{\prime}}{\eta^{\prime}} I_{\mathrm{NIR}} \sinh ^{2}\left(\frac{n}{2 \xi^{\prime} I_{\mathrm{NIR}}}\right) .
$$

365

366 Equation (12) provides a complete mathematical model 367 for the dynamics of ETM in the form of a nonlinear differ368 ential equation.

369 Section 5 below is dedicated to the study of the 370 equilibrium-state luminescence of ETM, which is the 371 equilibrium state of Eq. (12) $(\mathrm{d} n / \mathrm{d} t=0)$ :

$$
\begin{aligned}
372 \quad \frac{4 \xi}{\eta} I_{\mathrm{B}} \sinh ^{2}\left(\frac{n_{s}-n^{*}}{2 \xi I_{\mathrm{B}}}\right) & =\frac{4 \xi^{\prime}}{\eta^{\prime}} I_{\mathrm{NIR}} \sinh ^{2}\left(\frac{n^{*}}{2 \xi^{\prime} I_{\mathrm{NIR}}}\right), \\
{ }_{373}^{*} & =\alpha n^{*} I_{\mathrm{B}}+\beta n^{*} I_{\mathrm{NIR}} .
\end{aligned}
$$

374 In these equations, $n^{*}$ and $I_{\mathrm{O}}^{*}$ are the trapped-electron density and the intensity of the orange luminescence in 375 the equilibrium state, respectively. For any specified val- 376 ues of the blue light and NIR light intensities, Eq. (13) 377 can be solved for $n^{*}$ by minimizing the error function 378 er $f\left(n^{*}\right)$ :

$\operatorname{er} f\left(n^{*}\right)=\left\|\frac{4 \xi}{\eta} I_{\mathrm{B}} \sinh ^{2}\left(\frac{n_{s}-n^{*}}{2 \xi I_{\mathrm{B}}}\right)-\frac{4 \xi^{\prime}}{\eta^{\prime}} I_{\mathrm{NIR}} \sinh ^{2}\left(\frac{n^{*}}{2 \xi^{\prime} I_{\mathrm{NIR}}}\right)\right\|$.

In this equation, \|\| is the Euclidean norm. If one has the 381 value of $n^{*}$, the intensity of the orange light emission $I_{\mathrm{O}}^{*}$ is 382 computable from Eq. (14). By this methodology, one can 383 compute the value of $I_{\mathrm{O}}$ for a wide range of values of $I_{\mathrm{B}} \quad 384$ and $I_{\mathrm{NIR}}$ and prepare a diagram like the one shown in Fig. 385 10. In this two-dimensional diagram, which is called the 386 equilibrium-state plane (ESP) of the ETM, the contours of 387 constant orange light emission intensity are plotted as a 388 function of the blue light and NIR light intensity for a 389 reasonable range of variables on each axis. The numbers 390 on the contours are the output voltages of the photodetec- 391

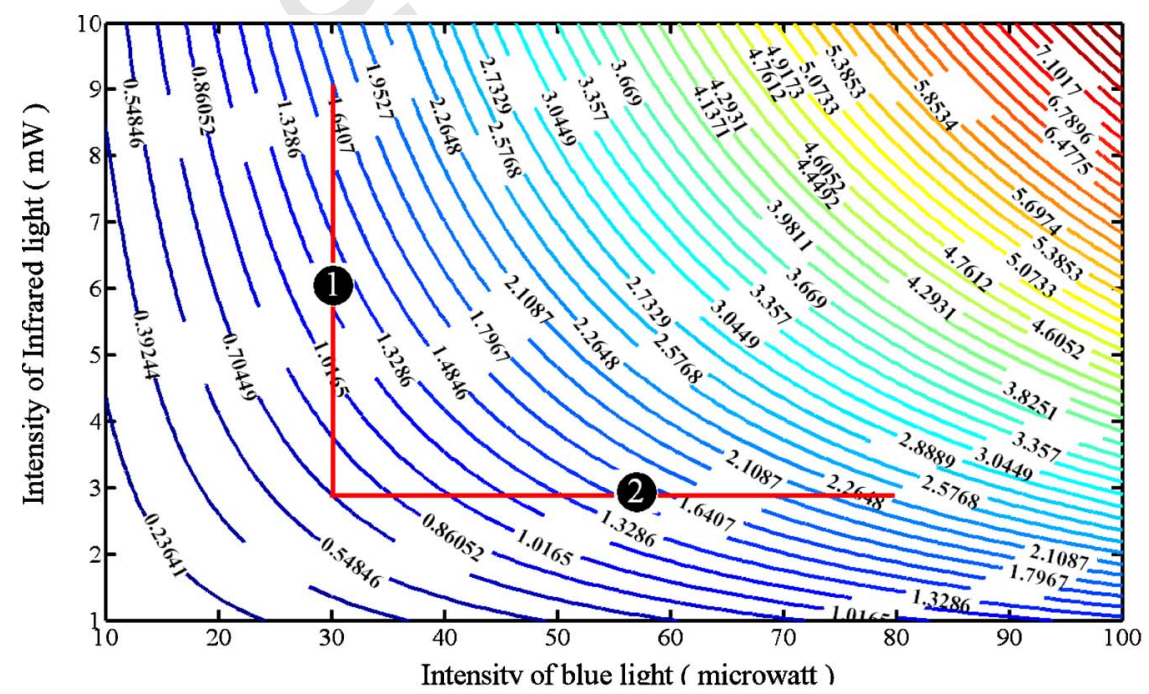

(a)

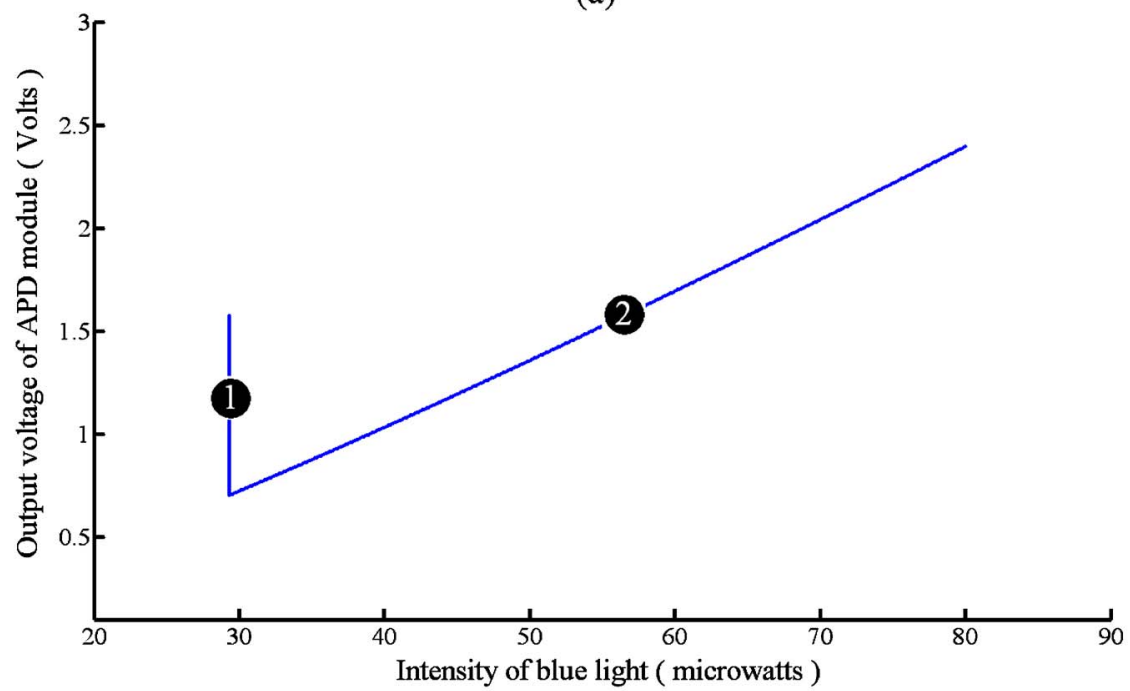

(b) 


\section{PROOF COPY [78556] 545708JOB}

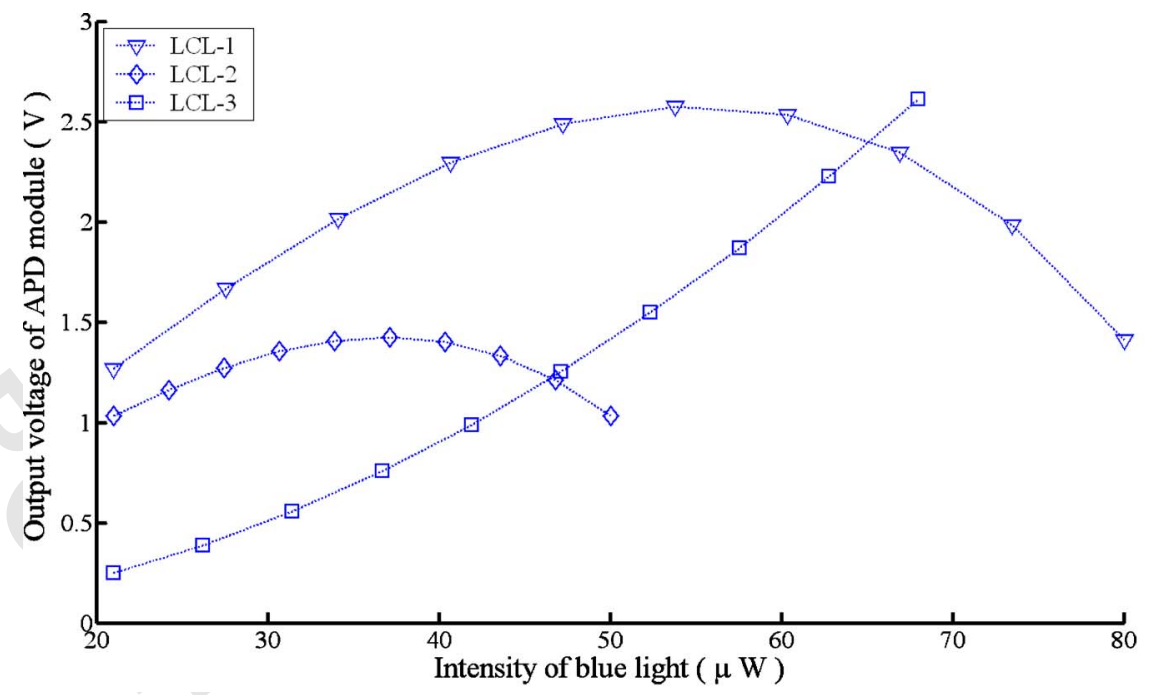

Fig. 16. (Color online) Experimental results. Along the first two LCLs, which have negative slopes, (see text) the luminescence of the material changes nonlinearly. Along the third LCL, which has positive slope, the luminescence of the material is quasi-linear.

392 tor that measures the intensity of the orange lumines393 cence. In Section 5, we will use this diagram as a graphic 394 tool to design highly nonlinear optical devices.

\section{5. EQUILIBRIUM-STATE LUMINESCENCE}

396 The significance of the equilibrium-state luminescence of 397 ETM for applications in optical signal processing becomes 398 clear by an example. Consider the simple optical arrange399 ment displayed in Fig. 11(a). A photodetector measures 400 the intensity of the light emitted from a light source and 401 passed through a passive optical device. Usually, the out402 put voltage of the photodetector is a linear function of the 403 intensity of illumination. However, in many applications 404 (e.g., optical realization of one-dimensional maps in non405 linear dynamics) we require a nonlinear behavior such as 406 the desired curve depicted in Fig. 11(b). Here, the inten407 sity of the detected light increases at first and decreases 408 when we pass a maximum point. To our knowledge such 409 an optical component is not available. In this section we show that the equilibrium-state luminescence of ETM can be controlled to exhibit such a nonlinear behavior. Based on the experimental results and the developed mathematical model, we then present an improved approach for realization and design of such nonlinear optical devices.

The behavior of ETM in the equilibrium state can be 416 predicted by using the ESP diagram that was introduced 417 in Section 4. We highlight the subtle utility of the ESP 418 diagram by investigating four sample points, $\mathrm{S}, 1,2$, and 419 3, in Fig. 10. Point $\mathrm{S}$ corresponds to the ETM panel being 420 exposed simultaneously to blue and NIR light with the 421 ETM in the equilibrium state. The intensity of the inci- 422 dent blue light and the NIR exposure are $50 \mu \mathrm{W}$ and 423 $4.35 \mathrm{~mW}$, respectively. The output voltage of the photode- 424 tector that measures the intensity of the orange light lu- 425 minescence is $\approx 1.8 \mathrm{~V}$.

Now, consider the case when the intensity of the blue 427 light is increased from $50 \mu \mathrm{W}$ to $60 \mu \mathrm{W}$, and in three suc- 428 ceeding steps, the intensity of the NIR light is changed to 429

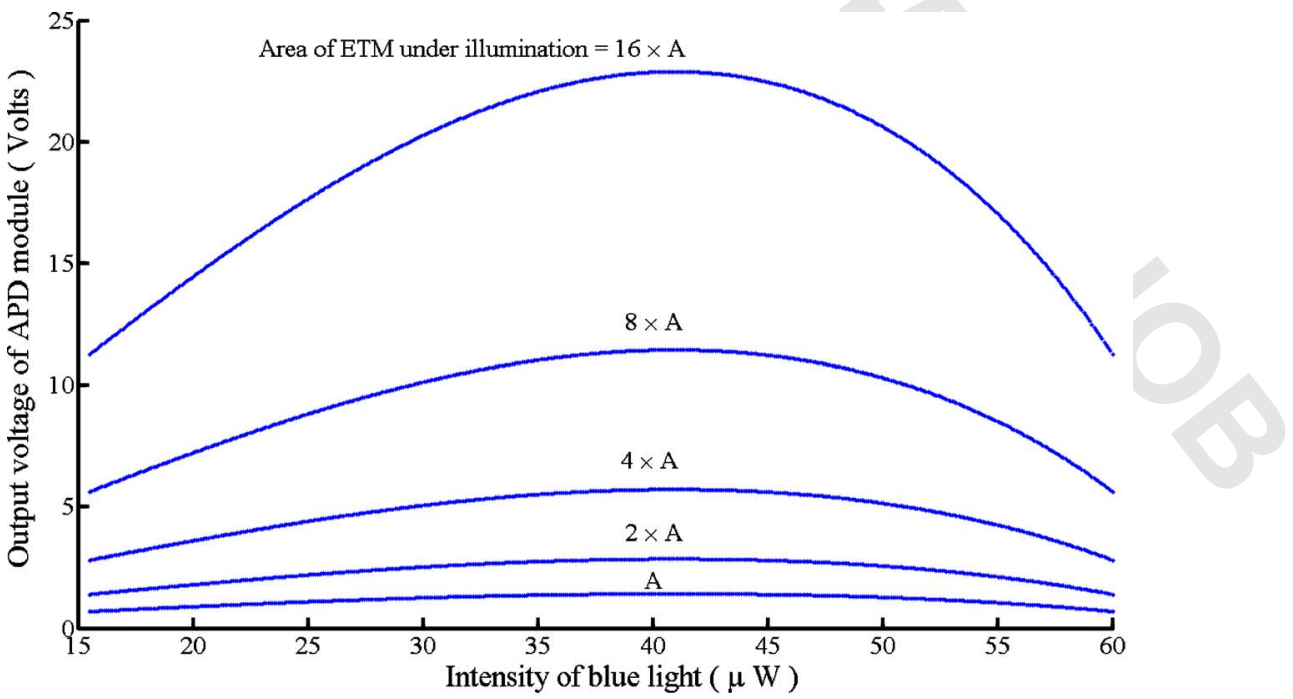

Fig. 17. (Color online) Effect of changing the area of the ETM under illumination. The nonlinear curves are related to the third LCL in Fig. 13(a). The area changes from $A=3 \mathrm{~mm}^{2}$ to $16 \times A$. 


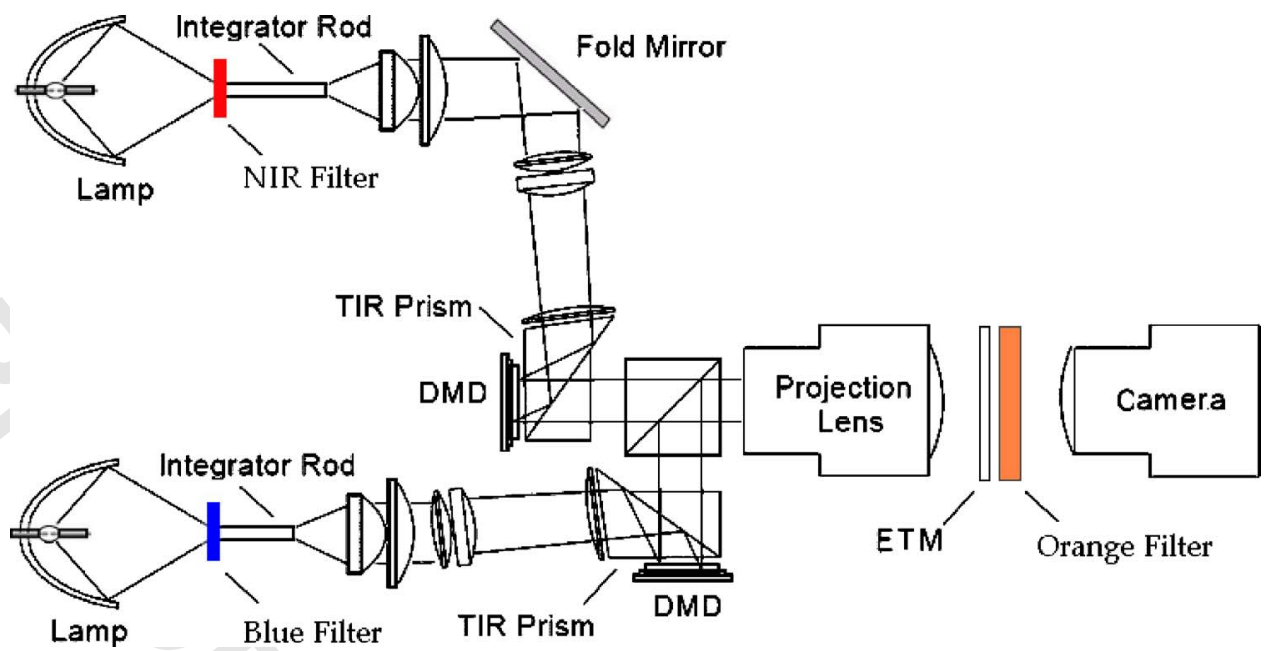

Fig. 18. (Color online) Simultaneous illumination of ETM panel with the combined beam of two DMD spatial light modulators. (TIR stands for total internal reflection.)

${ }^{430} 4 \mathrm{~mW}, 3.2 \mathrm{~mW}$, and $2.6 \mathrm{~mW}$. These new states are the 431 points 1,2 , and 3 , respectively, in Fig. 10. The output volt432 ages of the photodetector at these three points are 2.1, 4331.8 , and $1.5 \mathrm{~V}$ for points 1,2 , and 3 , respectively. As a re434 sult, by increasing the intensity of the blue light from 435 point $\mathrm{S}$ to points 1,2 , and 3 , depending on the intensity of 436 the NIR illumination, the intensity of the orange lumines437 cence increases, remains the same, or decreases, respec438 tively. Further investigation reveals that the intensity of 439 the emitted orange light monotonically increases along 440 the line $S \rightarrow 1$ and decreases along the line $S \rightarrow 3$. We have 441 recently shown that this kind of behavior can be used to 442 model the excitatory and inhibitory responses of the neu443 rons in optical realization of bio-inspired artificial neural 444 networks $[18,19]$.

445 Now consider the special case where the blue and NIR 446 light sources are linearly coupled. The linear coupling of 447 two light sources can be formulated by the equation

448

$$
\mu I_{\mathrm{B}}+\nu I_{\mathrm{NIR}}=\sigma,
$$

449 where $\mu, \nu$, and $\sigma$ are real numbers. These variables are 450 the key parameters in the design of the nonlinear optical 451 device. In this paper, the line expressed by Eq. (16) is 452 called the linear-coupling line (LCL). In the particular 453 case where the light sources are coupled linearly, the op454 tical device user can change the intensity of only one of 455 the light sources. For instance, in this paper, the blue 456 light source is the master source, and the NIR light 457 source traces the blue light source through Eq. (16). When 458 the two light sources are linearly coupled, those orange 459 light emission intensities along the corresponding LCL 460 only are achievable.

461 Two sample LCLs and their dynamic ranges over the 462 horizontal axis are illustrated in Fig. 12(a). The intensity 463 of the emitted orange light changes nonlinearly along 464 these two lines as shown in Fig. 12(b). A variety of non465 linear curves are achievable by changing the slope and 466 the location of a LCL in the ESP. For instance, the termi467 nation points of the first LCL in Fig. 12(a) are located on 468 the two different constant voltage contours. Therefore, 469 the intensities of the emitted orange light are different at these points. The second LCL has a different slope and dynamic range, and the corresponding nonlinear curve is quite different from the nonlinear curve of the first LCL; however, the termination points are both on the same contour. Therefore, the corresponding nonlinear orange light emission curve has equal intensities at the terminating points and the nonlinear curve is more symmetric.

These types of LCLs are suitable for the optical generation of nonlinear one-dimensional maps. Figure 13(a) displays two different LCLs with the same dynamic range. The corresponding nonlinear curves are illustrated in Fig. 13(b). In spite of similar dynamic ranges, these LCLs have different slopes and termination points. Hence, the corresponding nonlinear curves are different. A subtle difference between these two nonlinear curves is the speed of computation on these two LCLs. It should be remembered that all these curves are achievable in the equilibrium state of the ETM, which means that each time the intensities of the blue light and the NIR light are set, the user should wait for the equilibrium state to occur. The equilibrium is reached sooner for higher intensities of the blue and NIR light and larger density of the trapped electrons. The ETM reaches the equilibrium state faster along the second LCL of Fig. 13(a) than along the first LCL. Meanwhile, the intensity of orange luminescence is higher along the first LCL compared with the second one and the detected signal has a larger signal-to-noise ratio.

Now consider the case depicted in Fig. 14(a). In this figure, three parallel LCLs are shown in the ESP of the ETM. The corresponding orange light emission curves along these LCLs are shown in Fig. 14(b). The termination points of these three LCLs are placed on the same contour that represents the photodetector output voltage of $\approx 700 \mathrm{mV}$. These three LCLs are parallel to each other. Therefore, the parameters $\mu$ and $\nu$ in Eq. (16) are fixed, and the parameter $\sigma$ is the only variable. In nonlineardynamic applications, $\sigma$ can play the role of the bifurca- 506 tion parameter. By normalizing the dynamic ranges of 507 these lines, the developed nonlinear curves will look like 508 the curves that are used in the generation of a one- 509 dimensional map such as the logistic map in [20]. We 510 should mention that the quasi-linear behavior can also be 511

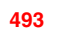
494 495 496 497 498 499 500 501 502 503 504

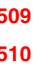
然

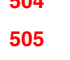
赵, (507 (1) 
512 generated by the equilibrium state of the ETM when the 513 LCLs are chosen appropriately. Figs. 15(a) and 15(b) illus514 trate two LCLs and the corresponding quasi-linear func515 tions, respectively.

516 In order to verify the above formulations an experiment 517 was carried out using the optical setup of Fig. 2. We chose 518 the following LCLs and measured the intensity of the or519 ange luminescence in the equilibrium state along each 520 line:

$521 \quad$ LCL-1: $-0.14 I_{\mathrm{B}}+I_{\mathrm{NIR}}=12.0$,
$522 \quad$ LCL-2: $-0.18 I_{\mathrm{B}}+I_{\mathrm{NIR}}=11.0$,
$523 \quad$ LCL-3: $+0.05 I_{\mathrm{B}}+I_{\mathrm{NIR}}=0.0$.

524 The results are shown in Fig. 16. In all these experi525 ments, the surface of the ETM panel is covered by a dark 526 optical mask with a small aperture. Thus, the area of the 527 ETM under illumination is fixed. On the other hand, one 528 can effectively take advantage of the area as a parameter 529 in the design of the nonlinear optical device. For a con530 stant blue and NIR light illumination, the level of the or531 ange light emission changes linearly as a function of the 532 area of the ETM under illumination. Figure 17 shows the 533 nonlinear curves developed by changing the illuminating 534 area for the third LCL in Fig. 14(a). We can use these 535 curves for the optical production of the one-dimensional 536 maps without any normalization.

537 One can easily change the area of ETM under illumi538 nation by using a spatial light modulator such as the 539 Texas Instruments digital micromirror device (DMD) [21]. 540 A sample optical setup is depicted in Fig. 18. A DMD chip 541 is a two-dimensional array of bistable programmable mi542 cromirrors. Two DMD modules in the setup provide the 543 blue light and the NIR illuminations by reflecting the 544 beam of two powerful light sources. By changing the num545 ber of mirrors, whose reflections expose the surface of the 546 ETM, the illuminating area can be changed. DMDs are 547 fast enough to adjust the area every few milliseconds, 548 which is shorter than the few hundred milliseconds re549 quired for an ETM to reach the equilibrium state.

\section{6. CONCLUSION}

551 We have introduced an improved mathematical model 552 that governs the dynamics of ETMs under blue light and 553 near-infrared illumination. The model describes the evo554 lution of an ETM's luminescence during charging, dis555 charging, simultaneous illumination, and in the equilib556 rium state. This model, which is established based on the 557 experimental results and takes into account some of the 558 previously neglected effects, can improve the applicability 559 of the ETM's unique dynamics, particularly in quantita560 tive applications such as optical signal processing. We 561 have also studied the nonlinear response of ETMs in the 562 equilibrium state. This nonlinear response has potential 563 applications in nonlinear optical signal processing and op564 tical implementation of one-dimensional maps. There are 565 occasions where collective processing in multiple one566 dimensional maps needs to be considered. An obvious ex567 ample of these applications are networks of one- dimensional maps [22-24]. The parallel computation carried out by such networks can be realized in a thin film 56 of this storage phosphor addressed suitably employing 570 DMD technology. Consequently, our mathematical model 571 would be an important tool for effective use of an ETM's 572 dynamics.

\section{ACKNOWLEDGMENTS}

This research was supported in part by Army Research 575 Office Multidisciplinary University Research Initiative 576 grant prime DAAD 19-01-0603 via Georgia Institute of 577 Technology subcontract E-18-677-64 and in part by Office 578 of Naval Research grant N00014-94-1-0931.

\section{REFERENCES AND NOTES}

1. J. Lindmayer, "A new erasable optical memory," Solid State 581 Technol. 31, 135-138 (1988).

2. J. Lindmayer, P. Goldmith, and K. Gross, "Electron- 583 trapping optical technology-memory's next generation," 584 Comput. Technol. Rev. 10, 37-42 (1990).

3. S. Jutamuli, G. Stori, J. Lindmayer, and W. Seiderman, 586 "Use of electron trapping materials in optical signal 587 processing. 1: parallel Boolean logic," Appl. Opt. 29, 588 4806-4811 (1990).

4. A. D. McAulay, J. Wang, and C. T. Ma, "Optical dynamic 590 matched filtering with electron trapping devices," in Real- 591 Time Signal Processing XI, J. P. Letellier, ed., Proc. SPIE 592 977, 271-276 (1988).

5. S. Jutamuli, G. Stori, J. Lindmayer, and W. Seiderman, 594 "Use of electron trapping materials in optical signal 595 processing. 2: two-dimensional associative memory," Appl. 596 Opt. 30, 2879-2884 (1991).

6. S. Jutamuli, G. Stori, J. Lindmayer, and W. Seiderman, 598 "Use of electron trapping materials in optical signal 599 processing. 3: modified Hopfield type neural networks," 600 Appl. Opt. 30, 1786-1790 (1991).

7. A. D. McAulay, J. Wang, and C. T. Ma, "Optical orthogonal 602 neural network associative memory with luminescence 603 rebroadcasting devices," in Proceedings of the IEEE 604 International Conference on Neural Networks (Proc. IEEE 605 1989) pp. 483-485.

8. F. Itoh, K. Kitayama, and Y. Tamura, "Optical outer- 607 product learning in a neural network using optically 608 stimulable phosphor," Opt. Lett. 15, 860-862 (1990). 609

9. S. Jutamulia, G. Storti, J. Lindmayer, and W. Seiderman, 610 "Optical information processing systems and 611 architectures," Proc. SPIE 1151, 83-xx (1990).

10. P. Soltani, D. Brower, and G. Storti, "Electron image tubes 613 and image intensifiers," Proc. SPIE 1243, 114-xxx (1990). 614

11. S. Keller, J. Mapes, and G. Cheroff, "Studies on some 615 infrared stimulable phosphors," Phys. Rev. 108, 663-676 616 (1957).

12. Z. Wen and N. Farhat, "Dynamics of electron trapping 618 materials for use in optoelectronic neurocomputing," Appl. 619 Opt. 32, 7251-7265 (1993). 620

13. Z. Wen, N. Farhat, "Electron trapping materials and 621 electron-beam-addressed electron trapping material 622 material devices: an improved model," Appl. Opt. 34, 623 5188-5198 (1995).

14. X. Yang, C. Y. Wrigley, and J. Lindmayer, "Three- 625 dimensional optical memory based on transparent electron 626 thin film," Proc. SPIE 1773, 413-422 (1992).

15. The electron-trapping material used for this investigation was furnished by the former Quantex Corporation, 629 Rockville, Maryland, USA.

16. Z. Hua, L. Salamanca-Riba, M. Wuttig, and P. K. Soltani, 631 "Temperature dependence of photoluminescence in 632 $\mathrm{SrS}: \mathrm{Eu}^{2+}, \mathrm{Sm}^{3+}$ thin films," J. Opt. Soc. Am. B 10, 633 1464-1469 (1993).

17. S. Boyd and L. Vandenberghe, Convex Optimization, 635 
PROOF COPY [78556] 545708JOB

R. Pashaie and N. H. Farhat

Vol. 24, No. 8/August 2007/J. Opt. Soc. Am. B

15

636

637 available online at http://www.stanford.edu/boyd/

638 18. R. Pashaie and N. H. Farhat, "Optical realization of the 639

640

$A Q:$

641 retinal ganglion receptive fields in electron-trapping material thin film," Proceedings of 32nd Northeast Bioengineering Conference, Easton, Penn., USA (2006).

42 19. R. Pashaie and N. H. Farhat, "Realization of receptive 643

644 fields with excitatory and inhibitory responses on the equilibrium-state luminescence of electron trapping

645

64620 . S. H. Strogatz, Nonlinear Dynamics and Chaos: With 647 Applications to Physics, Biology, Chemistry and 648
21. D. Dudley, W. M. Duncan, and J. Slaughter, "Emerging digital micromirror device (DMD) applications," Proc. SPIE 4985, 14-25 (2003).

22. K. Kaneko, "Overview of coupled map lattices," Chaos 2, 652 pp. 279-282 (1992).

23. N. H. Farhat, "Corticonics: the way to designing machines 654 with brain-like intelligence," Proc. SPIE 4109, 103-109 655 (2000).

24. N. H. Farhat, "Corticonic networks for higher-level 657

processing," Proc. 2nd IASTED, Feb. 23-25 (2004), pp. 658 256-262.
649

650

51

652

655

656

659 AQ:

PROOF COPY [78556] 545708JOB 


\section{AUTHOR QUERIES — 545708JOB}

\#1 Au: Claims of "new" deleted for legal reasons.

\#2 Au: Please define "Ip" in "Ip/mm"

\#3 Au: Check math in Eq. (4). Are parens and brackets as meant? Also in following equations?

\#4 Au: Please provide ending page number Refs. 9 and 10

\#5 Au: Please provide full dates of conference OR name of publishor of proceedings for Ref. 18.

\#6 Au: Ref. 24: Please provide full name of conference; spell out IASTED; give year of conf. and location.

\#7 Au: Fig. 10 caption: Is "unexpected" as meant? 